\title{
Four-point injection technique for lip augmentation
}

\author{
Ali Sahan ${ }^{1}$, Funda Tamer ${ }^{\circledR}$
}

\begin{abstract}
Introduction: Lip augmentation procedures with hyaluronic acid dermal fillers have become increasingly popular worldwide because full lips are often considered beautiful and youthful. The goal of a lip augmentation procedure is to create smooth lips with adequate volume and a natural appearance. Various techniques for lip augmentation have been utilized and described.

Methods: In the four-point injection technique, the lips were divided equally into right side and left side. Four entry points were made above the vermilion border for the upper lip and below the vermilion border for the lower lip. The filler was administered with a fanning technique through each entry point.

Results: Between January 2017 and November 2017, 50 female patients underwent a nonsurgical lip augmentation procedure with injectable fillers using this technique. Forty-five patients (90\%) reported that they were satisfied or extremely satisfied with their lip enhancement procedure. No serious complications were observed.

Conclusion: The advantages of this technique are reducing the risk of complications like erythema, edema, and vascular injuries, and providing easy access to injection sites.
\end{abstract}

Keywords: dermal filler, enhancement, hyaluronic acid, lip augmentation

Received: 26 November 2017 | Returned for modification: 19 January 2018 | Accepted: 25 January 2018

\section{Introduction}

Lip augmentation with hyaluronic acid dermal fillers is a minimally invasive procedure that has become increasingly popular in recent years (1). Lips become thinner with age due to a decrease in collagen and elastin (1). In elderly patients, filler injection into the lips aims to provide natural curves to the lips. However, the procedure is usually performed in younger patients to improve appearance by increasing lip volume (2). Hyaluronic acid fillers can also be used in the treatment of postoperative, accidental, and post-inflammatory scars on the lips or of asymmetric or incompetent lips (3). Herein, we describe the technique of lip augmentation with hyaluronic acid performed in cosmetic dermatology outpatient settings.

\section{Methods}

A topical antimicrobial agent (Octenisept) containing octenidine hydrochloride $0.1 \%$ and phenoxyethanol $2 \%$ was used to disinfect the skin. Both the upper and the lower lip were divided into symmetrical right and left sides by an imaginary vertical line through the center of the Cupid's bow. Four entry points were made using a 25-gauge needle. The entry points were located $5 \mathrm{~mm}$ above the vermilion border of the upper lip and $5 \mathrm{~mm}$ below the vermilion border of the lower lip.

Using a 27-gauge $25 \mathrm{~mm}$ cannula, $24 \mathrm{mg} / \mathrm{ml}$ hyaluronic acid + lidocaine $0.3 \%$ (Juvéderm Ultra 4) was injected into a divided part of the lip through the entry point. The filler was administered submucosally using a fanning technique. The direction of the cannula was changed two to four times, and filler was placed through these radial lines. Small boluses of 0.1 to $0.4 \mathrm{ml}$ were made at the injection area (Fig. 1). The same technique was applied to the other three parts of the lips.

Afterwards, a gentle massage of the injected areas was per- formed in order to maintain a smooth appearance. Patient satisfaction was evaluated with a four-point scale: disappointed, neutral, satisfied, extremely satisfied (4). The patients were advised to avoid hot showers, saunas, hot foods and beverages, and heavy exercise for the first 24 hours after filler injection. Touch-up treatment was administered one week after the initial treatment if required.

\section{Results}

Between January and November 2017, 50 female patients underwent a nonsurgical lip augmentation procedure with injectable fillers using the four-point technique described above. Patients' medical records were reviewed retrospectively. The mean age was 28.1 years, with a range of 18 to 46 years. The patients had not been treated for lip enhancement in the prior 12 months. The exclusion criteria were having an autoimmune disease or keloidprone skin, allergy to hyaluronic acid, and current treatment with anticoagulants.

A total volume of approximately 0.4 to $1.6 \mathrm{ml}$ hyaluronic acid filler was injected into the lips. The results were evaluated immediately after the procedure and again 1 week later (Figs. 1-5). A total volume of 0.1 to $0.3 \mathrm{ml}$ of hyaluronic acid was administered to 32 patients $(64 \%)$ to correct asymmetry 1 week after the initial injection. Forty-five patients (90\%) reported that they were satisfied or extremely satisfied with their lip enhancement procedure. Three patients (6\%) were neutral and two patients (4\%) reported being disappointed with the results. The latter two had unrealistic expectations for lip augmentation and were not completely pleased with the appearance of their lips.

Minimal pain and a small amount of redness were observed at the needle entry points. However, no bruising, bleeding, swelling, itching, or serious complications like infection, necrosis, discoloration, lumps, or foreign body granuloma occurred. 


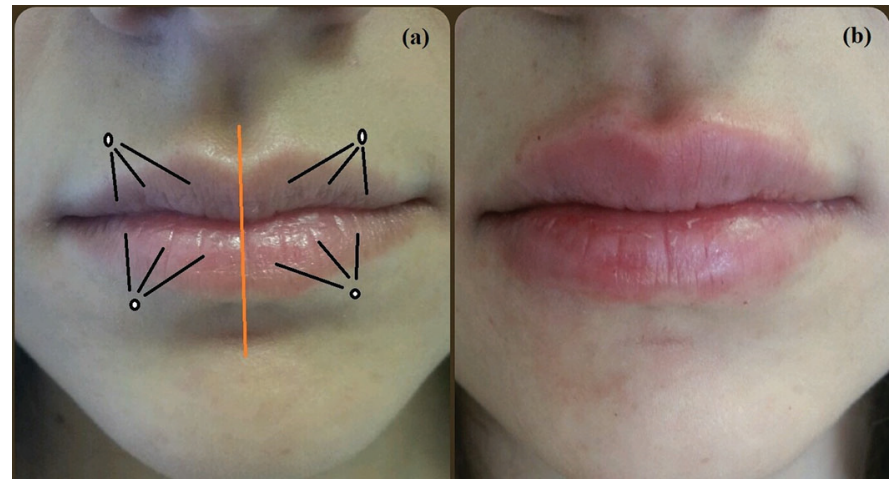

Figure 1 | (a) Four-point injection technique. The lips were divided vertically into two equal parts. Four entry points were made $5 \mathrm{~mm}$ above/below the center of the vermilion border of each side. The filler was injected submucosally using a cannula with a fanning technique (b) post-treatment view of the patient.

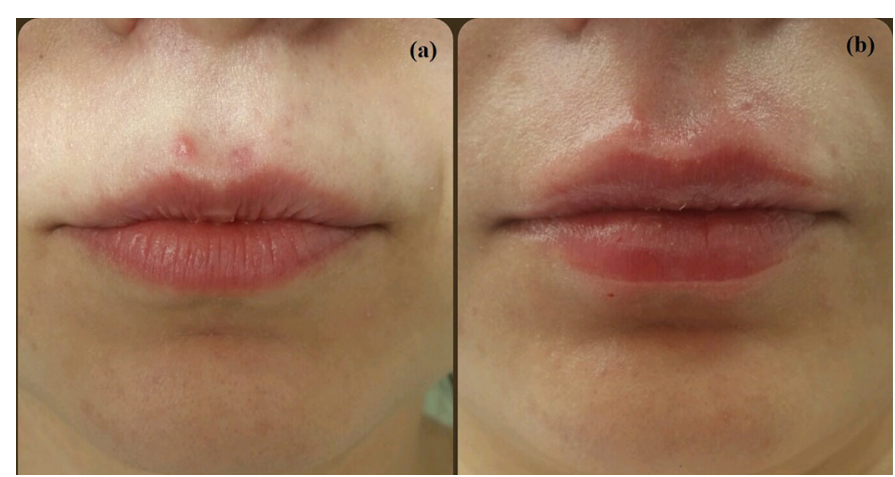

Figure 2 | (a) Before and (b) immediately after the lip augmentation procedure.

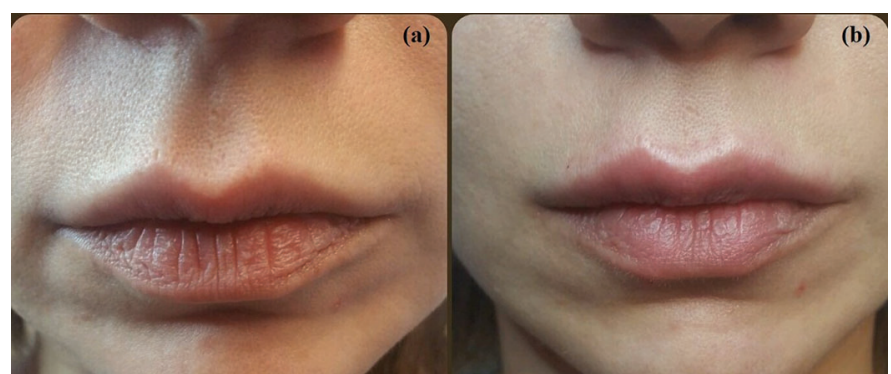

Figure 3 | (a) Before and (b) immediately after the lip augmentation procedure.

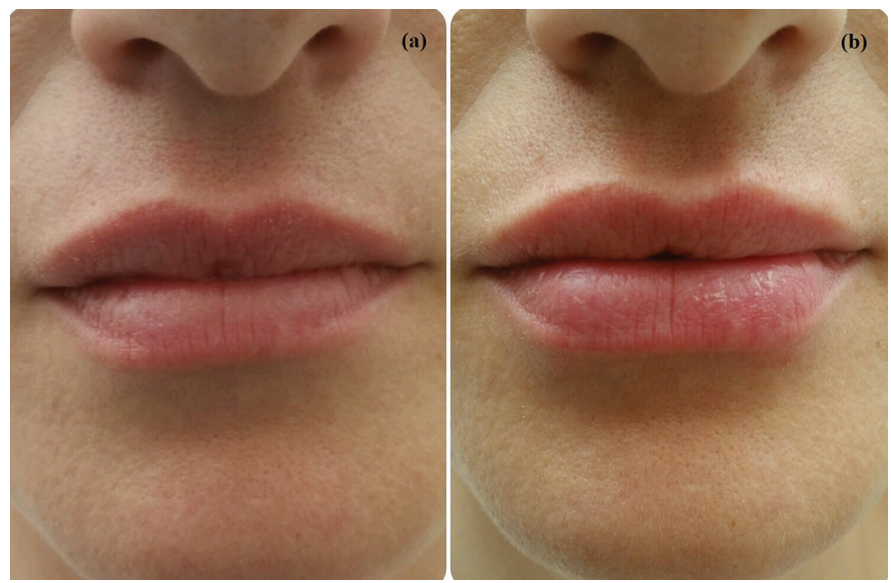

Figure 4 | (a) Before and (b) immediately after the lip augmentation procedure.

\section{Discussion}

Lip augmentation with improved techniques has gained popularity because full lips are often associated with beauty and youth (5). The goal of a lip augmentation procedure is to create smooth lips with adequate volume and a well-defined vermilion border (6). There is no single way to achieve the intended results. How-

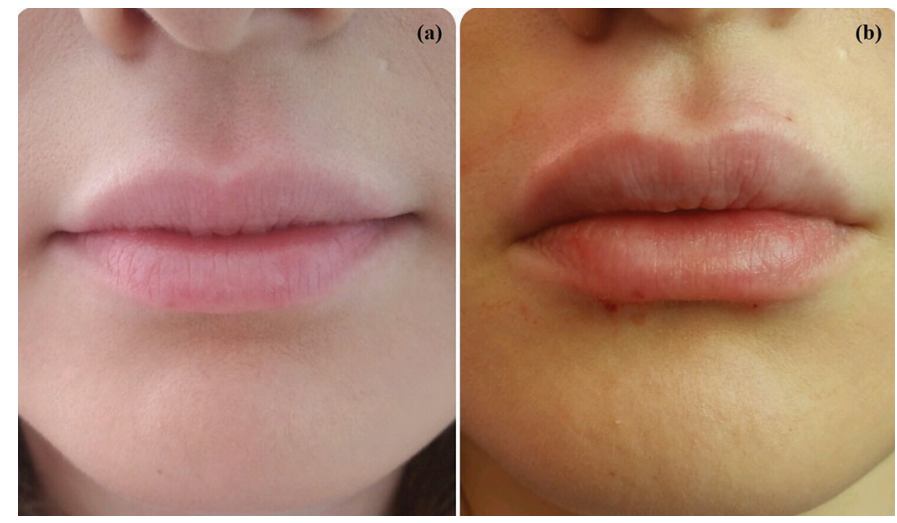

Figure 5 | (a) Before and (b) immediately after the lip augmentation procedure.

ever, anatomical knowledge and the use of appropriate products and techniques are essential for a natural appearance (7).

Lip enhancement techniques are classified into surgical and nonsurgical procedures (8). One surgical procedure, the triple V-Y augmentation technique, is performed without using dermal fillers or implants and aims to expand vermilion by advancing the labial mucosa forward (8). Other options for surgical lip augmentation include direct lip lift and subnasal lip lift procedures (9). Surgical lip implants and autologous fat transfer are used only rarely (10).

The injection of dermal fillers is the most popular nonsurgical procedure performed to increase the volume and improve the shape of the lips (11). Semi-permanent dermal fillers-such as calcium hydroxylapatite and poly-L-lactic acid-and permanent fillers are not preferred for lip augmentation because they have an increased risk of irregularity and nodule formation. Hyaluronic acid fillers are the most commonly used products for lip enhancement (10).

Various injection techniques have been described for lip augmentation. Needle injections are recommended for superficial administration to avoid vascular injury. Nevertheless, cannulas can be used safely in deep injections (12). The depth of the injection plays a crucial role in achieving good aesthetic and functional outcomes (13). Blandford et al. compared the injection of hyaluronic acid into the upper lip vermilion border to needle and microcannula injection techniques. They indicated that using a microcannula provides a more uniform administration of hyaluronic acid to the intramuscular zone (13).

The injection type options include serial puncture, linear threading, cross-hatching, and fanning (10). Border injection, marginal injection, and eversion technique are the preferred techniques for defining lip margins. The border injection technique uses injections along the vermilion border, the marginal injection technique uses injections starting with the dry-wet junction or the skin-mucosa junction and proceeding to the inferior part of the upper lip and the superior part of the lower lip, and the eversion technique uses the administration of filler into the submucosa within the oral vestibule. Furthermore, fanning injections through the vermilion border of the Cupid's bow create a midline labial tubercle. Serial transverse or longitudinal injections add volume to both the upper and lower lips. The towering technique, which uses deep perpendicular injections with a needle along the vermilion lines, can also be performed to create voluminous lips (12).

Surek et al. described a no-touch technique for lip enhancement that does not violate the mucosa. The entry point is $5 \mathrm{~mm}$ lateral from the oral commissure. Dermal fillers are injected by ei- 
ther a needle or a cannula beneath the white roll to improve the lip profile. However, this technique administers filler into the submucosal space, which is inferior to injecting it into the red roll (14).

Sarnoff et al. recommend a six-step technique to achieve a natural look. This technique uses approximately 12 injections of around 0.1 cc hyaluronic acid each to the upper and lower lips. The technique consists of stages: creating the philtrum columns and Cupid's bow, defining the vermilion-cutaneous junction of the upper lip, creating lower lip tubercles, supporting the oral commissures, and filling the upper part of the nasolabial folds (15).

We had previously administered multiple injections of hyaluronic acid fillers with a needle through the dry and wet mucosal lips. Then we began using a technique that requires a cannula to be inserted through the lateral side of the vermilion border, and we also performed multiple punctures with a needle through the vermilion border of the lips. Most recently, we used the four-point injection technique detailed above. To the best of our knowledge, four-point injection technique for lip augmentation has not been described previously. In this technique, fillers are placed with a cannula via four entry points. Using a cannula minimizes the risk of complications like erythema, edema, ecchymosis, and vascular injuries. Moreover, the four entry points provide easy access to injection sites despite the use of a cannula.

\section{References}

1. Hilton S, Sattler G, Berg AK, Samuelson U, Wong C. Randomized, evaluatorblinded study comparing safety and effect of two hyaluronic acid gels for lips enhancement. Dermatol Surg. 2018;44:261-9.

2. Bass LS. Injectable filler techniques for facial rejuvenation, volumization, and augmentation. Facial Plast Surg Clin North Am. 2015;23:479-88.

3. Stolic D, Jankovic M, Draskovic M, Georgiev S, Stolic M. The surgical lips deformity corrected with hyaluronic fillers: a case report. Open Access Maced J Med Sci. 2015;3:423-5.

4. Dierickx CC, Mazer JM, Sand M, Koenig S, Arigon V. Safety, tolerance, and patient satisfaction with noninvasive cryolipolysis. Dermatol Surg. 2013;39:1209-16.

5. Byrne PJ, Hilger PA. Lip augmentation. Facial Plast Surg. 2004;20:31-8.

6. Clymer MA. Evolution in techniques: lip augmentation. Facial Plast Surg. 2007;23:21-6.

7. Sarnoff DS, Saini R, Gotkin RH. Comparison of filling agents for lip augmentation. Aesthet Surg J. 2008;28:556-63.

8. Obradovic B, Obradovic M. Triple V-Y vermilion augmentation of the upper lip. Craniofac Surg. 2015;26:e736-8.
9. Maloney BP, Truswell W 4 th, Waldman SR. Lip augmentation: discussion and debate. Facial Plast Surg Clin North Am. 2012;20:327-46.

10. Mannino GN, Lipner SR. Current concepts in lip augmentation. Cutis. 2016;98: 325-9.

11. Landau M. Lip augmentation and rejuvenation using Dermicol-P35 30G: personal experiences from my clinic. Aesthet Surg J. 2009;29:S12-5.

12. Tansatit T, Apinuntrum P, Phetudom T. Cadaveric assessment of lip injections: locating the serious threats. Aesthetic Plast Surg. 2017;41:430-40.

13. Blandford AD, Hwang CJ, Young J, Barnes AC, Plesec TP, Perry JD. Microanatomical location of hyaluronic acid gel following injection of the upper lip vermillion border: comparison of needle and microcannula injection technique. Ophthalmic Plast Reconstr Surg. 2018;34:296-9.

14. Surek CC, Guisantes E, Schnarr K, Jelks G, Beut J. "No-touch" technique for lip enhancement. Plast Reconstr Surg. 2016;138:603e-13e.

15. Sarnoff DS, Gotkin RH. Six steps to the "perfect” lip. J Drugs Dermatol. 2012;11: 1081-8. 